\title{
CONSTRUCCIÓN Y PROGRAMACIÓN DE SENSORES DE BAJO COSTO COMPATIBLES CON EL KIT DE ROBÓTICA LEGO MINDSTORMS NXT
}

Fernando Martínez Santa

Magister en Ingeniería Electrónica y de Computadores

Docente planta de la Universidad Distrital Francisco José de Caldas fmartinezs@udistrital.edu.co Bogotá, Colombia

\section{Fernando Rivera Sánchez}

Tecnólogo en Electricidad Universidad Distrital Francisco José de Caldas

hfriveras@correo.udistrital.edu.co Bogotá, Colombia

\section{William Alexander Sáenz}

Tecnólogo en Electricidad Universidad Distrital Francisco José de Caldas wasaenzr@correo.udistrial.edu.co Bogotá, Colombia

Tipo: Artículo reporte de caso

Fecha de Recepción: Febrero 27 de 2013 Fecha de Aceptación: Mayo 2 de 2013

\section{CONSTRUCTION AND PROGRAMMING OF LOW-COST SENSORS COMPATIBLE WITH THE LEGO MIND- STORMS ROBOTICS KIT NXT}

\begin{abstract}
This paper briefly presents the key concepts related to the implementation and construction of low-cost sensors for the LEGO Mindstorms robotics kit NXT. The kit mainly consists of a smart block with four input ports that capture the information coming from different sensors. These can be scheduled from as LeJOS software, and LabVIEW BRICXCC among others. This document shows the process necessary for the design and implementation of libraries in these languages, in order to operate low-cost sensors built, and thus provide guidelines and tools for user and student to implement SBC1 their own.. Key points are discussed in terms of connection and adaptation of the NXT SBC and its differences with respect to the original sensors and approved kit.
\end{abstract}

Key words: accelerometer, distance, hall effect, Mindstorms NXT, sensors, temperature.

\section{RESUMEN}

El presente artículo expone de forma breve los conceptos claves relacionados con la implementación y construcción de sensores de bajo costo para el kit de robótica LEGO Mindstorms NXT. El kit principalmente consta de un bloque inteligente con cuatro puertos de entrada que permiten capturar la información que proviene de distintos sensores. Estos se pueden programar a partir de software como LeJOS, BRICXCC y LabVIEW entre otros. En este documento se muestra el proceso necesario para el diseño e implementación de librerías en estos lenguajes, con el fin de poner en funcionamiento los sensores de bajo costo construidos, y de esta manera brindar pautas y herramientas necesarias para que el usuario y estudiante pueda implementar sus propios S.B.C1. Se exponen puntos claves en cuanto a la conexión y adaptación de los S.B.C al NXT y sus diferencias con respecto a los sensores originales del kit y los homologados.

Palabras claves: acelerómetro, distancia, efecto hall, Mindstorms NXT, sensores, temperatura. 


\section{INTRODUCCIÓN}

Desde ya hace algunos años el kit de robótica LEGO Mindstorms NXT, ha sido una valiosa herramienta en el aprendizaje y en la investigación de la robótica a nivel universitario. Existen actualmente gran variedad de sensores compatibles con este kit en el mercado local e internacional, los cuales son construidos por la empresa LEGO o por las otras empresas homologadas, teniendo estos la desventaja de ser muy costosos. El objetivo principal de este trabajo es crear una guía de construcción de sensores de bajo costo compatibles con el kit LEGO Mindstorms NXT, con el fin de que los estudiantes interesados en replicar o utilizar dichos sensores, no tengan limitantes económicas a la hora de desarrollar sus proyectos.

Se destacan tres elementos importantes en este documento: el análisis, diseño y construcción de cuatro sensores aplicados al kit de robótica LEGO Mindstorms NXT. Se plantea la explicación de cómo se pueden construir sensores de bajo costo para el kit de robótica.

Los S.B.C NXT UD, son sensores construidos con materia prima de bajo costo que funcionan como herramientas de apoyo para el kit de robótica LEGO Mindstorms NXT, con el objetivo de que el usuario pueda importar, organizar, exportar y compartir librerías y/o programas con completa funcionalidad y compatibilidad con éste.

\section{DESARROLLO}

El componente principal del kit de robótica LEGO Mindstorms NXT (figura 1) es un bloque inteligente. Con éste se puede obtener información de hasta cuatro sensores y controlar tres motores, a través de cables RJ12.

El bloque tiene una pantalla LCD monocromática de $100 \times 64$ píxeles y cuatro botones que se pueden utilizar para navegar con una interfaz de usuario con menús jerárquicos (figura 2). Cuenta con un procesador de 32-bit ARM7, $256 \mathrm{~kb}$ de memoria flash, $64 \mathrm{~KB}$ de RAM, un microcontrolador AVR de 8-bits, y soporte para bluetooth. También cuenta con un altavoz y puede reproducir archivos de sonido a velocidades de muestreo de hasta $8 \mathrm{kHz}$. La energía es suministrada por la baterías es de 6 pilas AA (1,5 V cada una) en la versión de consumo del equipo y por una batería recargable de litio con su respectivo cargador, en la versión educativa [1].

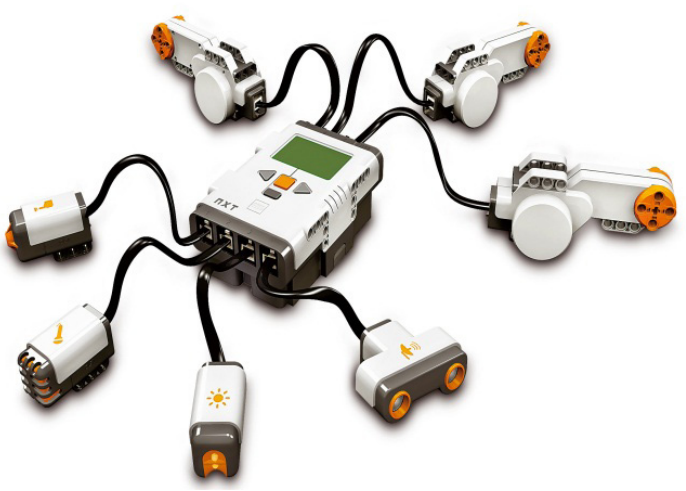

Figura 1. Kit de robótica LEGO Mindstroms NXT.

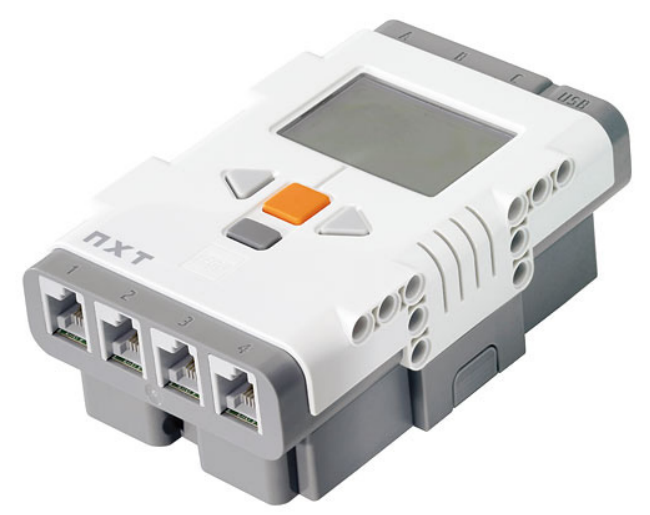

Figura 2. Bloque programable NXT.

A continuación de exponen los puntos clave para la construcción y programación de los S.B.C NXT UD.

\subsection{Pines de conexión del cable NXT}

Para la construcción de los S.B.C NXT UD, fundamentalmente se tuvo en cuenta en primera instancia los pines de conexión que hay en cada uno de los cuatros puertos de salida que tiene el bloque programable NXT. Cada uno de estos puertos está conformado por una entrada análoga y dos entradas/salidas digitales que mane- 
jan el protocolo de comunicación I2C, una es la señal de datos (SDA) y la segunda es la señal de reloj (SCL), además tiene salida de tensión y dos tierras, en la tabla 1 se observa los pines de conexión según el color de los cables del NXT.

Tabla 1. Pines de conexión del cable NXT.

\begin{tabular}{|c|c|}
\hline Color & PIN \\
\hline Azul & SDA \\
\hline Amarillo & SCL \\
\hline Verde & VD \\
\hline Rojo & GND1 \\
\hline Negro & GND2 \\
\hline Blanco & OUT \\
\cline { 2 - 2 } &
\end{tabular}

\subsection{S.B.C de temperatura NXT UD}

S.B.C de temperatura U.D NXT principalmente está compuesto por un sensor de temperatura LM35, el cual tiene una precisión calibrada de $1^{\circ} \mathrm{C}$. Su rango de medición abarca desde $-55^{\circ} \mathrm{C}$ hasta $150^{\circ} \mathrm{C}$. La salida es lineal y cada grado centígrado equivale a $10 \mathrm{mV}$ [2].

Sus características más relevantes son:

- Esta calibrado directamente en grados Celsius.

- La tensión de salida es proporcional a la temperatura.

- Tiene una precisión garantizada de $0.5^{\circ} \mathrm{C}$ a $25^{\circ} \mathrm{C}$.

- Debido a su baja corriente de alimentación se produce un efecto de auto calentamiento muy reducido.

- Bajo costo.

- Baja impedancia de salida, 0,1 $\Omega$ de carga a $1 \mathrm{~mA}[2]$.

\subsubsection{Construcción}

Para la construcción del S.B.C NXT UD de temperatura, fundamentalmente se tuvieron en cuenta los pines de conexión con respecto al bloque NXT, información que especificada en la tabla 1, para éste caso se explica la conexión del LM35 el cual se muestra en la figura 3.
Luego de identificar los pines de conexión del sensor al NXT, se realiza un circuito de adaptación a éste, el cual incluye una resistencia de $220 \Omega$, ésta se coloca con el objetivo de garantizar en la entrada análoga del NXT una corriente de $18 \mathrm{~mA}$ [3], la cual cumple la función de activar el conversor análogo digital al no realizar este proceso el conversor A/D no funciona correctamente y no realizará la discretización de datos de voltaje del LM35.

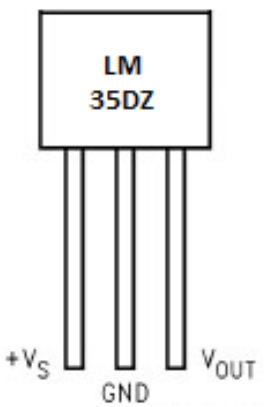

Figura 3. Conexión del LM35 [2].

Lo anterior se realiza con el fin de que el NXT logre procesar correctamente los datos, la resistencia de $220 \Omega$ se calculó mediante la ecuación (1).

$$
\begin{aligned}
R_{A / D} & =\frac{V_{\text {out }}}{18} \frac{[\mathrm{V}]}{[\mathrm{mA}]} \\
R_{A / D} & =\frac{4}{18} \frac{[\mathrm{V}]}{[\mathrm{mA}]} \\
R_{A / D} & =220[\Omega]
\end{aligned}
$$

En la figura 4 se muestra el esquema de conexión del sensor al cable NXT.

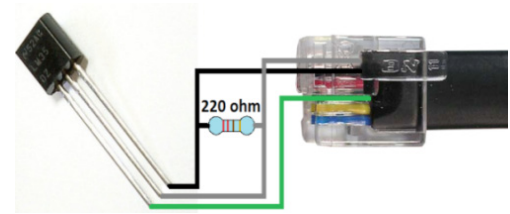

Figura 4. Esquema de conexión del LM35 al NXT.

\subsubsection{Programación}

Con respecto a la programación y adaptación al NXT, se realiza la identificación gráfica general a partir de un diagrama de flujo que se muestra 
en la figura 5, el cual expone los puntos principales que se llevan a cabo para desarrollar el algoritmo necesario, con la finalidad de realizar el proceso, tratamiento y recolección de datos de registro para capturar la medición adecua$\mathrm{da}$, ofreciendo al usuario garantías de exactitud máxima que permite este sensor.

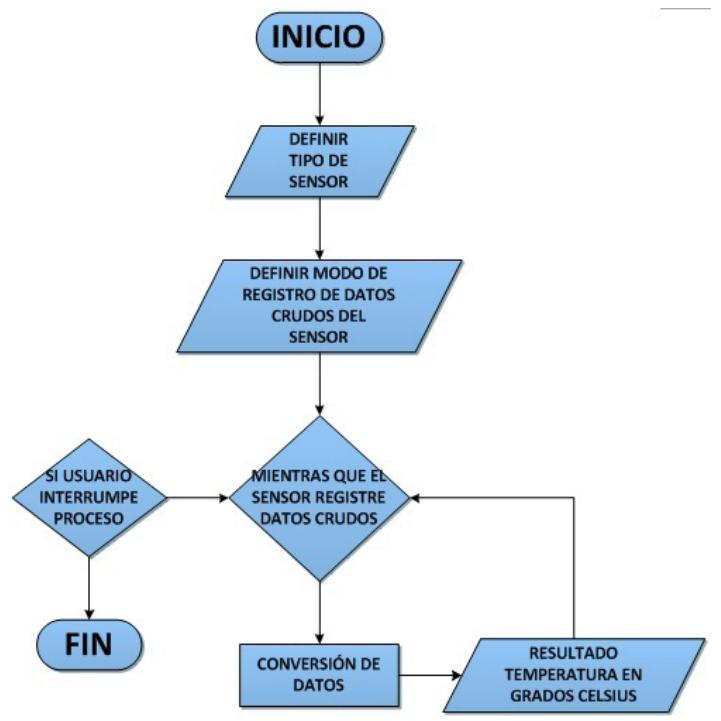

Figura 5. Diagrama de flujo S.B.C de temperatura NXT UD.

Es importante mencionar que el algoritmo mostrado anteriormente en el diagrama de flujo es general con respecto a los tres lenguajes de programación mencionados (LeJOS, NXC, lenguaje $G$ ) ya que la estructura de funcionamiento es la misma.

La fórmula para hallar el valor real de la temperatura se determina mediante la ecuación (2).

$$
\text { Temperatura }\left[{ }^{\circ} \mathrm{C}\right]=R A W \frac{5[V]}{10\left[\frac{m V}{{ }^{\circ} \mathrm{C}}\right] * 1024}
$$

Donde:

RAW: Son los valores crudos del sensor 5[V]: Tensión máxima análogica a convertir $10\left[\mathrm{mV} /{ }^{\circ} \mathrm{C}\right]$ : Tensión de salida del sensor por ${ }^{\circ} \mathrm{C}$ 1024: Es un número que sale de 0 a 1023, que equivalen a los 10 bits de resolución del conversor análogo digital del NXT.

\subsection{S.B.C de efecto hall NXT UD}

El S.B.C de efecto hall U.D NXT principalmente está compuesto por un sensor de efecto hall OH090U que opera con una amplia gama de tensiones de alimentación. Se caracteriza por permitir la conducción directa de más de 7 cargas TTL o cualquier familia de la lógica estándar, utilizando fuentes de alimentación que van desde 4,5 a 24 V. Gran estabilidad en los datos de registro con cambios de las frecuencias DC desde más de $200 \mathrm{kHz}$, en este caso la amplitud de salida siempre es constante.

Respuesta booleana, "0-Ausencia de campo; 1-Presencia de campo", esta característica hace que este sensor sea ideal para aplicaciones en las operaciones de conmutación sin contacto [4].

Sus características más relevantes son:

- Respuesta booleana, "0-Ausencia de campo; 1-Presencia de campo".

- Diseñado para las operaciones de conmutación sin contacto.

- Funciona en todo el rango amplio de tensiones de alimentación (4,5 V a 24 V).

- Funciona con excelente estabilidad de temperatura en ambientes y entornos.

- Con capacidad de hasta 7 cargas TTL [4].

\subsubsection{Construcción}

Para su construcción se tomó en cuenta la caracterización de la tabla 1 , y su esquema de conexión a partir de un circuito de adaptación al NXT el cual incluye una resistencia de $820 \Omega$ entre VD y OUT, como se muestra en la figura $6[4]$. 
Por consiguiente la conexión del sensor al cable del NXT se demuestra en la figura 7.

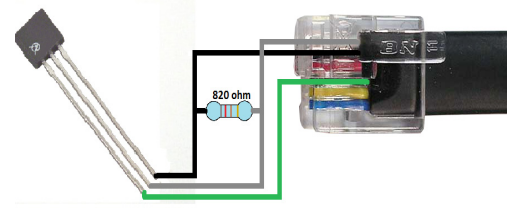

Figura 7. Conexión del OH090U al NXT .

\subsubsection{Programación}

En la figura 8 se muestra un diagrama de flujo que describe el algoritmo necesario para la implementación del OH090U al NXT, esto se realiza con el fin de exponer de forma general la manera en la cual se puede programar dicho sensor.

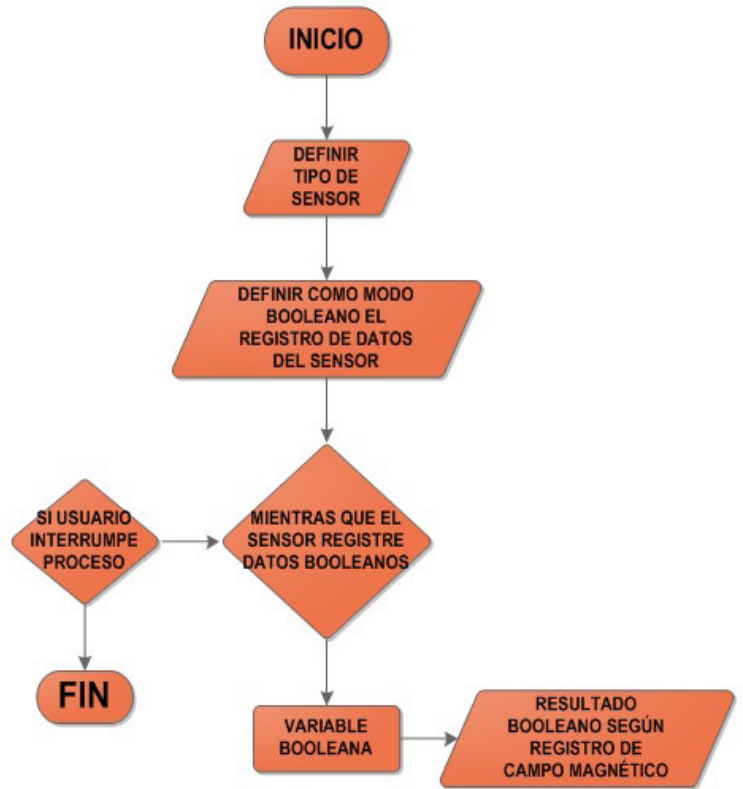

Figura 8. Diagrama de flujo S.B.C de efecto hall NXT UD.

\subsection{S.B.C de ultrasonido NXT UD}

S.B.C de ultrasonido U.D NXT principalmente está compuesto por un sensor SFR02, el cual es un telémetro transductor de ultrasonidos en una pequeña huella de PCB. Debido a que éste utiliza un único transductor tanto para la transmisión y recepción, el intervalo mínimo de datos de registro es más alto que en otros rangos de transductores duales. El rango de medición mínima varía entre $17-18 \mathrm{~cm}$ (7 pulgadas) y en un día caluroso y fresco hasta alrededor de 15$16 \mathrm{~cm}$ ( 6 pulgadas). Al igual que todos los telémetros, en éste se puede medir en microsegundos, centímetros o en pulgadas.

Hay dos modos de funcionamiento para el SRF02. El modo I2C y el modo Serial. Para éste caso el SFR02 se conectó en modo I2C por facilidad y compatibilidad con el ladrillo programable NXT [5].

Sus características más relevantes son:

- Esta calibrado directamente en centímetros.

- Usado para medir distancia en un rango de $15 \mathrm{~cm}$ a $6 \mathrm{~m}$ [5].

\subsubsection{Construcción}

Teniendo como referencia de nuevo la tabla1, el esquema de conexión del SFR02 de muestra en la figura 9.

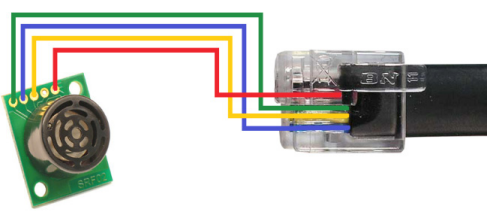

Figura 9. Conexión del SFR02 al NXT.

Como se evidenciar en la figura 9 la conexión del sensor al cable NXT es directa, por ésta razón no hay que realizar circuito de adaptación al ladrillo NXT ya que al realizar las pruebas necesarias con el programa de adquisición de datos no hubo la necesidad de emplear resistencias u otro elemento pasivo en el circuito. La comunicación del SFR02 se puede realizar de dos maneras: por medio de comunicación serial y por I2C, para éste caso se realizó por medio de I2C ya que el NXT por defecto ya viene con dicho protocolo de comunicación.

\subsubsection{Programación}

En la figura 10 se muestra un diagrama de flujo que describe el algoritmo necesario para la implementación del SFR02 al NXT, esto se realiza 
con el fin de exponer de forma general la manera en la cual se puede programar dicho sensor.

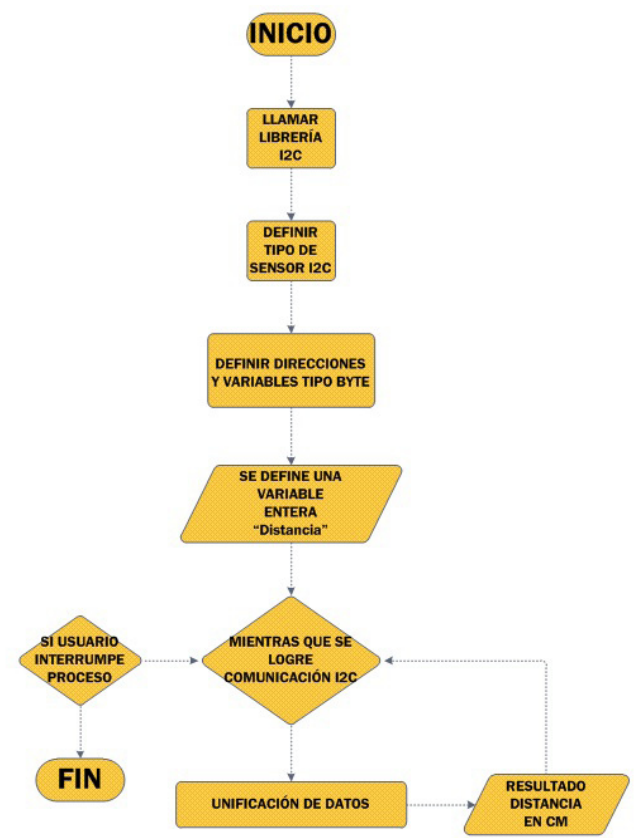

Figura 10. Diagrama de flujo S.B.C de ultrasonido NXT UD.

La ecuación (3) halla el valor de la distancia registrada.

Dis $\tan$ cia $=$ byte alto $* 256+$ byte bajo $[\mathrm{cm}](3)$

Donde:

byte alto: 8 bits mas significativos.

byte bajo: 8 bits menos significativos. 256: Factor de corrimiento de 8 bits.

\subsection{S.B.C acelerómetro NXT UD}

El S.B.C acelerómetro U.D NXT principalmente está compuesto por una tarjeta con MMA7361LT el cual es un acelerómetro de 3 ejes de $\pm 1.5 \mathrm{~g}$, bajo consumo de corriente ( 400 $\mu \mathrm{A})$, alta sensibilidad ( $800 \mathrm{mV} / \mathrm{g}$ a $1.5 \mathrm{~g})$, voltaje de operación 2.2 - 3.6V [6].

Sus características más relevantes son:
- $\quad$ Rango de medición de $\pm 1.5 \mathrm{~g}$.

- Bajo consumo de corriente: $400 \mu \mathrm{A}$.

- Bajo voltaje de operación: $2.2 \mathrm{~V}-3.6 \mathrm{~V}$.

- Alta sensibilidad (800 mV/g a $1.5 \mathrm{~g}$ ).

- Rápido tiempo de registro de datos $(0.5 \mathrm{~ms}$ de tiempo de respuesta).

- Acondicionamiento de señal con filtro pasabajo (filtro caracterizado por permitir el paso de las frecuencias más bajas y atenuar las frecuencias más altas).

- Diseño robusto, con alta resistencia al impacto [6].

\subsubsection{Construcción}

Para la adaptación de la tarjeta MMA361L al NXT se identificaron los pines de conexión con respecto a la tabla 1 , posteriormente se identificaron dos inconvenientes:

- El sensor necesita una alimentación de $3,3 \mathrm{~V}$.

- Los pines de conexión análoga del cable NXT no son suficientes para adquirir los datos del sensor.

Para esta situación se estudiaron métodos para solucionar las contrariedades presentadas en la construcción y adaptación del sensor al NXT, se llegaron a las siguientes conclusiones:

Para solucionar el problema del exceso de caída de tensión del NXT al sensor, se insertaron dos resistencias de $1200 \Omega$ en serie con la alimentación del sensor, forzando de esta forma la caída de tensión del sensor a 3,3V.

Finalmente, con respecto a la necesidad de adquirir todos los datos de registro del sensor, se programó el PIC 16F818 con el fin de ofrecer más pines de conexión al NXT (figura 11), para garantizar la comunicación I2C entre éstos, para esto se conectaron dos resistencias de Pull-Up, con el objetivo de aumentar la caída de tensión entre los pines digitales del NXT asegurando de esta manera el protocolo de comunicación y el registro adecuado de datos revelados por el sensor. 


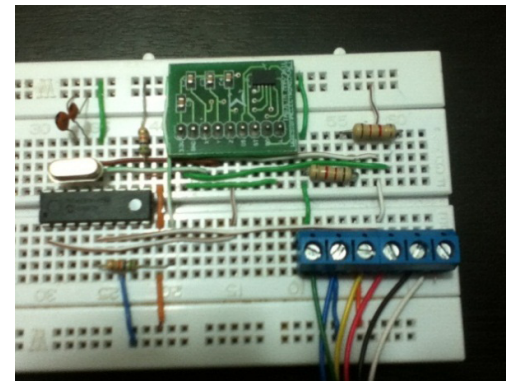

Figura 11. Conexión la tarjeta MMA7361L al NXT.

\subsubsection{Programación}

En la figura 13 y 14 se muestran los diagramas de flujo que describen los algoritmos necesarios para la implementación de la tarjeta MMA7361L y el proceso que se debe llevar a cabo en el PIC 16F818 para lograr la comunicación I2C al NXT, esto se realiza con el fin de exponer de forma general la manera en la cual se puede programar dicho sensor.

En la figura 12 se expone la manera en la cual se realiza la comunicación entre el sensor y el bloque NXT.

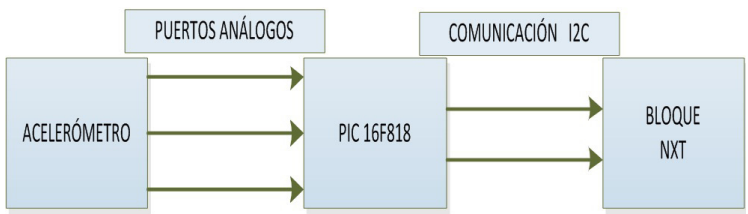

Figura 12. Comunicación entre el acelerómetro y el bloque NXT.

Para hallar el valor real de la inclinación y aceleración a partir de los datos del sensor se utiliza la ecuación (4).

$$
\text { Aceleración }=\frac{V_{\text {out }}-V_{\text {offset }}}{\left(\frac{\Delta V}{\Delta g}\right)}\left[\frac{m}{s^{2}}\right]
$$

Donde:

$\mathrm{V}_{\text {out }}$ : Tensión promedio de los ejes en $0 \mathrm{~g}$. $\mathrm{V}_{\text {offset }}$ :Tensión de salida de los ejes. $\Delta \mathrm{V} / \Delta \mathrm{g}$ : Sensibilidad del sensor.

9,8: Gravedad de la tierra en $\mathrm{m} / \mathrm{s}^{2}$.

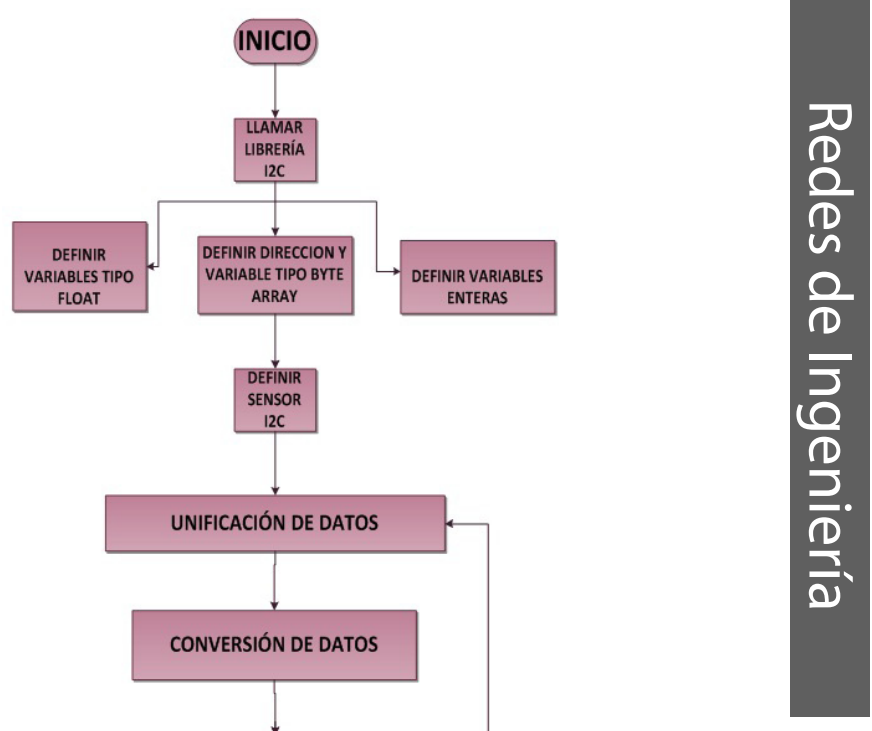

Figura 13. Diagrama de flujo S.B.C acelerómetro NXT UD.

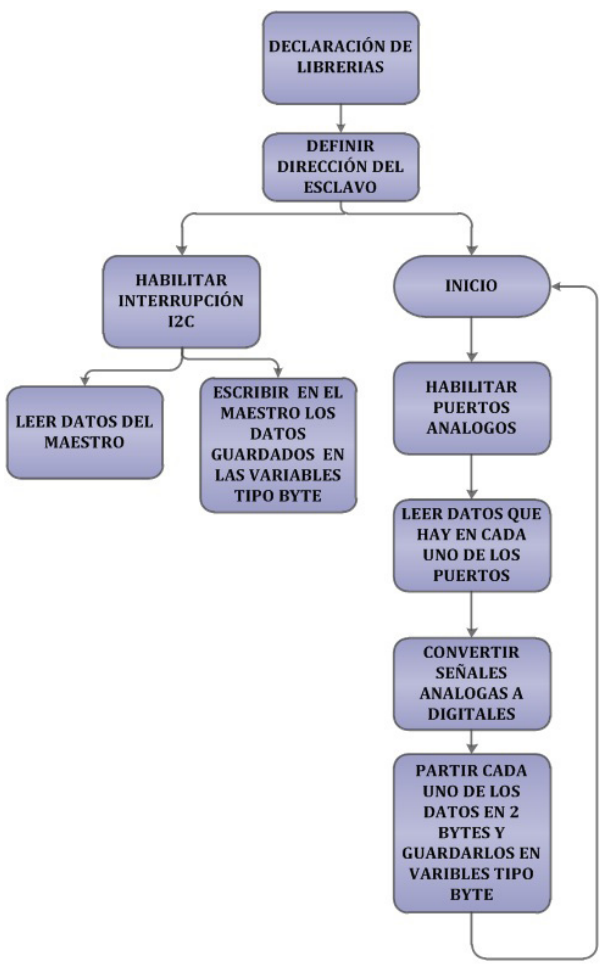

Figura 14. Diagrama de flujo para el PIC 16F818 (Comunicación I2C al NXT) 


\section{RESULTADOS}

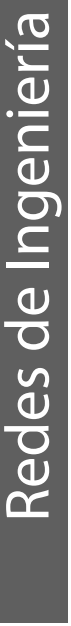

A continuación se presentan tablas comparativas de características y precios entre los sensores S.B.C, los originales del kit y los de otras empresas avaladas por la empresa LEGO.
En la tabla 2 se realiza la comparación para los sensores de temperatura, en la tabla 3 para los sensores de efecto hall, en la tabla 4 para los sensores de ultrasonido y en la tabla 5 para los acelerometros.

Tabla 2. Comparación de sensores de temperatura.

\begin{tabular}{|l|c|c|c|c|}
\hline \multicolumn{5}{|c|}{ SENSOR DE TEMPERATURA } \\
\hline \multicolumn{1}{|c|}{ Empresa } & S.B.C NXT UD & Dexter Industries & Hitechnic & Vernier \\
\hline Rango de medida $\left[{ }^{\circ} \mathrm{C}\right]$ & $0 \mathrm{~A} 150$ & $-50 \mathrm{~A} 150$ & $-20 \mathrm{~A} 120$ & $-40 \mathrm{~A} 135$ \\
\hline Precisión $\left[{ }^{\circ} \mathrm{C}\right]$ & $+/-0.9$ & $+/-0,5$ & $+/-0,5$ & $+/-0,5$ \\
\hline Sensibilidad $[\mathrm{mV} / \mathrm{g}]$ & 800 & -- & -- & -- \\
\hline No linealidad $\left[{ }^{\circ} \mathrm{C}\right]$ & $+/-0.2$ & -- & -- & -- \\
\hline Error relativo $[\%]$ & $-3,6 \%[2]$ & -- & -- & -- \\
\hline Precio $[\$ \mathrm{M} / \mathrm{C}][3]$ & $\$ 27.311$ & $\$ 183.000$ & $\$ 196.000$ & $\$ 233.000$ \\
\hline
\end{tabular}

Tabla 3. Comparación de sensores de efecto hall.

\begin{tabular}{|l|c|c|c|}
\hline \multicolumn{4}{|c|}{ SENSOR DE EFECTO HALL } \\
\hline \multicolumn{1}{|c|}{ Empresa } & S.B.C NXT UD & Hitechnic & Vernier \\
\hline Rango de medida [Gauss] & 90 A 180 & -- & 0,3 a 6,4 \\
\hline Precisión [Gauss] & -- & -- & $+/-0,32$ \\
\hline Precio [\$ M/C] [3] & $\$ 34.300$ & $\$ 196.000$ & $\$ 220.000$ \\
\hline
\end{tabular}

Tabla 4. Comparación de sensores de ultrasonido.

\begin{tabular}{|l|c|c|}
\hline \multicolumn{3}{|c|}{ SENSOR DE DISTANCIA O ULTRASONIDO } \\
\hline Empresa & Rango de medida[cm] & Precio [\$ M/C] [3] \\
\hline S.B.C NXT UD & 15 a 600 & $\$ 69.400$ \\
\hline Mindsensors & 30 a 150 & $\$ 185.000$ \\
\hline Mindsensors & 10 a 80 & $\$ 185.000$ \\
\hline Mindsensors & 6 a 30 & $\$ 185.000$ \\
\hline Hitechnic & 0 a 20 & $\$ 172.000$ \\
\hline NXT & 0 a 255 & \\
\hline
\end{tabular}

Tabla 5. Comparación de sensores acelerómetros.

\begin{tabular}{|l|c|c|c|c|c|}
\hline \multicolumn{7}{|c|}{ SENSOR ACELERÓMETRO } \\
\hline \multicolumn{1}{|c|}{ Empresa } & S.B.C NXT UD & Dexter Industries & Hitechnic & Mindsensors & Vernier \\
\hline Rango de medida [g] & $+/-1.5$ & $+/-2,+/-4 \mathrm{y}+/-8$ & $+/-2$ & -- & $+/-5$ \\
\hline Sensibilidad [mV/g] & 800 & -- & 5 & $1,5,2,4 \mathrm{y} 6$ & 50 \\
\hline Precio [\$ M/C] [3] & $\$ 50.080$ & $\$ 245.000$ & $\$ 196.000$ & $\$ 186.000$ & $\$ 268.000$ \\
\hline
\end{tabular}




\subsection{Relación calidad/costo}

La relación calidad costo se realizó con el fin de determinar cuáles son los beneficios por cada peso que obtendría el usuario al elegir cualquiera de los sensores, ya sean los originales de LEGO, los de empresas homologadas o los S.B.C NXT UD.

La relación se calculó a partir ecuación (5).

$$
\frac{\text { Calidad }}{\text { Costo }}=\frac{\text { SPAN }+K^{*} \text { Precisión }}{\text { Precio }}\left[\frac{{ }^{\circ} \mathrm{C}}{\$}\right]
$$

El SPAN y la precisión son seleccionadas como características de calidad, y "K" es el factor de ajuste para equiparar el peso de las dos características en la ecuación. Esta última es calculada a partir del promedio del SPAN y de la precisión de los sensores de todas las empresas incluyendo el S.B.C NXT UD.

En la tabla 6 se especifica la relación calidad costo a partir de la ecuación (5) para el sensor de temperatura.

Tabla 6. Costo/beneficio sensor de temperatura.

\begin{tabular}{|l|c|c|c|c|}
\hline \multicolumn{5}{|c|}{ COSTO/BENEFICIO TEMPERATURA } \\
\hline \multicolumn{1}{|c|}{ Empresa } & S.B.C NXT UD & Dexter Industries & Hitechnic & Vernier \\
\hline Precisión & 0,9 & 0,5 & 0,5 & 0,5 \\
\hline Span & 150 & 200 & 140 & 175 \\
\hline Precio [M/C] & $\$ 27.311$ & $\$ 183.000$ & $\$ 196.000$ & $\$ 233.000$ \\
\hline Costo/Beneficio & $1,10 \%$ & $0,15 \%$ & $0,11 \%$ & $0,11 \%$ \\
\hline
\end{tabular}

\subsection{Análisis de resultados}

Visiblemente resulta más económico construir los sensores a partir de las condiciones expuestas, que comprarlos originales del kit o por empresas homologadas por la empresa LEGO, debido a que el usuario ahorraría en promedio lo siguiente:

- Si el usuario desea adquirir el S.B.C NXT UD de temperatura, ahorraría $86,61 \%$ con respecto al precio promedio de la competencia.

- $\quad$ Si el usuario desea adquirir el S.B.C NXT UD de efecto hall, ahorraría $83,51 \%$ con respecto al precio promedio de la competencia.

- $\quad$ Si el usuario desea adquirir el S.B.C NXT UD distancia o ultrasonido, ahorraría 60,43\% con respecto al precio promedio de la competencia.

- $\quad$ Si el usuario desea adquirir el S.B.C NXT UD acelerómetro, ahorraría $77,62 \%$ con respecto al precio promedio de la competencia.
Con respecto a la implementación de los sensores al NXT, se demostraron algoritmos en diferentes tipos de lenguajes de programación, verificando de esta forma la potencia y la versatilidad que posee el NXT en cuanto a su adaptación a diferentes instrumentos electrónicos.

Deduciendo que algunos usuarios pueden suponer que los sensores tienen poca eficiencia a raíz de su bajo precio, se demostró a través de tablas comparativas que las diferencias entre los sensores originales y los de bajo costo son mínimas con respecto a su eficiencia y funcionamiento.

Para facilidad del usuario y/o estudiante se diseñaron librerías con el objetivo de ofrecer el acceso básico a los S.B.C NXT UD. La información necesaria del uso y la implementación de las librerías de los S.B.C NXT UD en los diferentes lenguajes que se encuentran especificados en un manual de usuario en formato digital.

A partir de los resultados obtenidos en la tabla 6 , se puede concluir que es más aconsejable para el usuario adquirir o construir los S.B.C 
NXT UD ya que su relación calidad costo es mayor que 1, esto indica que sus beneficios son mayores con respecto al precio de adquisición, en cambio los sensores originales de LEGO y los construidos por empresas homologadas, su relación calidad costo está por debajo de 1, esto sugiere que su precio es más alto con respecto al beneficio que presta.

\section{PERSPECTIVAS}

Con respecto a proyectos futuros se presentan algunos sensores que pueden ser adaptados al kit de robótica NXT.

- Sensor de corriente.

- Sensor brújula magnética.

- Sensor de humedad.

- Sensor de ángulo.

Por otro lado es importante resaltar algunos puntos claves de los S.B.C construidos.

Para el sensor de temperatura se puede limitar el paso de corriente conectando un amplificador, que además de cortar corrientes negativas, amplifica el voltaje de su entrada, con el

Referencias Bibliográficas

[1] LEGO Group, What is NXT?. Enero 2010, [en línea]. Consultado en mayo 10 de 2012, disponible en: http://mindstorms. lego.com/enus/whatisnxt/default.aspx

[2] National Semiconductors, LM35 Precision centigrade temperature sensors. Noviembre de 2000, [en línea]. Consultado en octubre 2 de 2012, disponible en: https://www.national.com/ ds/LM/LM35.pdf

[3] LEGO Group, LEGO Mindstorms NXTHardware Developer Kit. Enero 2006, [en línea]. Consultado en abril 5 de 2012, disponible en: http://ebookbrowse. com/lego-mindstorms-nxt-hardwaredeveloper-kit-pdf-d1384528.

[4] OPTEK Technologies, Hallogic Halleffect Sensors. Octubre de 2006, [en objetivo de incrementar el rango de medición del sensor [7].

A la hora de conectar el LM35 al NXT aparecen limitaciones en cuanto a los valores negativos de temperatura [8]. Estas limitaciones están provocadas por el efecto de carga de la resistencia de $220 \Omega$ con la salida del sensor. Esto produce que el sensor se vea afectado y se eviten las tensiones negativas, para solucionar esto al circuito se le puede adicionar un cambiador de nivel negativo que desplaza la señal de tensión a valores negativos, con el objetivo de que el LM35 pueda considerar valores por debajo de $0^{\circ} \mathrm{C}$.

Con respecto al sensor acelerómetro se puede realizar un selector de sensibilidad, con el fin de utilizar totalmente la funcionalidad del sensor.

El usuario puede calibrar el sensor de ultrasonido para que su resultado de en pulgadas o microsegundos a partir de cambiar la dirección de lectura del I2C por las presentadas en el datasheet del mismo. línea]. Consultado en octubre 12 de 2012 , disponible en: http://datasheet.octopart. com/OH090U-TT-datasheet8638342.pdf

[5] Robot electronics, SRF02 Ultrasonic range finder Technical Specification. Febrero de 2009, [en línea]. Consultado en junio 2 de 2012, disponible en: http://www.robotelectronics.co.uk/htm/ srf02tech.htm

[6] Freescale Semiconductor, Technical Data $\pm 1.5 \mathrm{~g}, \pm 6 \mathrm{~g}$ Three Axis Low-g Micromachined Accelerometer. Abril de 2008, [en línea]. Consultado en junio 2 de 2012, disponible en:http://www. freescale.com/files/sensors/doc/data sheet/MMA7361L.pdf

[7] Xavier Viñolo Arcos, Sistema sensor para navegación en vehículos autónomos. 
Enero de 2010, [en línea]. Consultado en junio 22 de 2012, disponible en: http://upcommons.upc.edu/pfc/ bitstream/2099.1/9377/1/memoria.pdf

[8] M. Gasperi, P. Hurbain, Extreme NXT: Extending the LEGO MINDSTORMS
NXT to the Next Level. Enero 2009, [en línea]. Consultado en junio 10 de 2012, disponible en: http://brweb.haltonrc. edu.on.ca/202204/ICE4/Resources/ ExtremeNXT.pdf. 